\title{
The role of matrix metalloproteinase 14 polymorphisms in susceptibility to intervertebral disc degeneration in the Chinese Han population
}

\author{
Jianfeng Zhang ${ }^{1,2}$, Xiujiang Sun ${ }^{3}$, Jing Liư , Jianqing Liư ${ }^{2}$ Binghua Shen ${ }^{3}$, Lin $\mathrm{Nie}^{1}$
}

${ }^{1}$ Department of Orthopedics, Qilu Hospital of Shandong University, JiNan City, Shandong Province, China

${ }^{2}$ Department of Spine Surgery, Yantaishan Hospital, YanTai City, Shandong Province, China

${ }^{3}$ Department of Bone and Joint, Yantaishan Hospital, YanTai City, Shandong Province, China

${ }^{4}$ Department of Basic Research, Occupation College of Yantai, YanTai City, Shandong Province, China

Submitted: 19 June 2013

Accepted: 18 August 2013

Arch Med Sci 2015; 11, 4: 801-806

DOI: 10.5114/aoms.2015.53301

Copyright @ 2015 Termedia \& Banach

\section{Abstract}

Introduction: Matrix metalloproteinase 14 (MMP14) plays an important role in the pathophysiology of intervertebral disc degeneration (IVDD). The present study aimed to determine whether two single nucleotide polymorphisms $(-378 \mathrm{~T} / \mathrm{C}$ and $-364 \mathrm{G} / \mathrm{T})$ of MMP14 were associated with the risk and severity of IVDD in the Chinese Han population.

Material and methods: A total of 908 patients with IVDD and 906 healthy controls were enrolled in this study. The grade of disc degeneration was determined according to Schneiderman's classification for magnetic resonance imaging. The polymorphisms of MMP14 were genotyped using polymerase chain reaction and direct sequencing.

Results: The genotype distribution of $-364 \mathrm{G} / \mathrm{T}$ did not show a significant difference between IVDD patients and healthy controls. The frequencies of the -378 T/C and CC genotypes were significantly lower among IVDD patients compared with healthy controls $(p<0.001)$; unconditional logistic regression analysis revealed that the $\mathrm{CT}$ and $\mathrm{CC}$ genotypes were significantly associated with a decreased risk of IVDD compared with the TT genotype $(p<0.001)$. Patients with IVDD showed significantly higher frequencies of the T allele at $-378 \mathrm{~T} / \mathrm{C}$ than healthy controls $(p<0.001)$. In addition, the -375 CC genotype, as well as the C allele, was associated with lower degenerative grades of IVDD compared with the TT genotype and the T allele, respectively (both $p<0.001$ ).

Conclusions: The $-378 \mathrm{~T} / \mathrm{C}$ polymorphism of MMP14 may be associated with the risk and severity of IVDD in the Chinese Han population. It shows potential to become a biomarker to predict risk and severity of IVDD.

Key words: polymorphism, MMP14, $-378 \mathrm{~T} / \mathrm{C},-364 \mathrm{G} / \mathrm{T}$, intervertebral disc degeneration.

\section{Introduction}

Intervertebral disc degeneration (IVDD) is the major cause of low back pain. Low back pain is a major cause of disability and it substantially

\author{
Corresponding author: \\ Prof. Lin Nie \\ Department of Orthopedics \\ Qilu Hospital \\ of Shandong University \\ No. 107 Wenhua West Road \\ JiNan City 250012 \\ Shandong Province, China \\ Phone: +8653182169114 \\ Fax: +86 53182169114 \\ E-mail: linnie55@163.com
}


contributes to healthcare costs [1, 2]. The etiology and pathogenesis of IVDD are still unclear. Causes of IVDD are likely to be both genetic and environmental, but are probably also related to wear accumulating over a lifetime of normal daily use [3]. Recently, a series of genetic factors have been proved to play important roles in the development of IVDD. Polymorphisms in a number of genes, such as aggrecan [4, 5], interleukin 1 [6], vitamin D receptor [7], and matrix metalloproteinase [8, 9], are reported to be associated with an increased risk of IVDD [10].

The development of disc degeneration is a complex, multi-stage process in which the degradation of the disc matrix is one of the key steps. A group of matrix degrading enzymes, the matrix metalloproteinase (MMPs), are assumed to play a critical role in the excessive breakdown of the extracellular matrix (ECM) during disc degeneration. Matrix metalloproteinase 14, an important member of the MMP family, is of particular importance to intervertebral disc homeostasis [11, 12]. Increased expression and activity of MMP14 has been documented in disc tissue with degenerative lesions [13].

Matrix metalloproteinase 14 , also called membrane type 1 MMP (MT1-MMP), usually forms a tri-molecular complex with tissue inhibitor of metalloproteinase-2 (TIMP-2) and proMMP-2 on the cell surface; proMMP-2 can be activated and released at a low concentration of TIMP-2 to mediate the ECM degradation [14-18]. Matrix metalloproteinase 14 is able to be a pericellular collagenase against ECM components directly [14]. In a variety of cell types, MMP14 is employed to act on the surrounding environment and to promote tissue remodeling, invasion, and metastasis by catalyzing pericellular collagenolysis [12]. In this way, it provides advantageous circumstance for cells proliferating in a constrained three-dimensional collagen or fibrin microenvironment.

Transcription of MT1-MMP is regulated by two enhancers, the Sp1 binding site and regulatory region 1 (RR1). Recently, two single nucleotide polymorphisms (SNPs) at the MT1-MMP promoter were identified in close vicinity to the RR1: -364 G/T (rs1003349: G > T) and -378 T/C (rs1004030: $\mathrm{T}>\mathrm{C}$ ). In vitro transcriptional analysis with luciferase receptor constructs of human MMP14 promoter demonstrates that occurrence of the SNP variant $-378 \mathrm{C}$ leads to strong inhibition of nuclear protein binding to the RR1 reducing its enhancer function, while $-364 T$ does not [11].

Although there is no direct evidence to suggest that MMP14 expression is related to the pathophysiology of IVDD, there are reports suggesting increased MMP-2 activity during intervertebral disc degeneration is correlated with MMP14 levels
$[13,19]$, and the demonstration of the presence of MMP14 in the IVDD and its increased production during degeneration could have important implications [20]. Expression and polymorphism of MMP2 have been proved to correlate with the risk and severity of lumbar disc degeneration in the Chinese Han population [21].

To date, an association between the MMP14 SNPS and IVDD has not well been investigated. In this study, we performed a case-control study to explore the possible role of MMP14 SNPs in determining the susceptibility and severity of IVDD in a Chinese cohort. Genetic studies are crucial for understanding the mechanism of the degeneration. This genetic information could eventually be used as a predictive marker for determining a patient's risk for symptomatic IVDD.

\section{Material and methods}

\section{Patients}

A total of 908 patients with IVDD were enrolled in this study. They were all symptomatic patients with surgically or radiologically proven IVDD. Patients with synovial cysts, spondylolisthesis, spinal tumor, spondylosis, trauma and inflammatory disease were excluded. The grade of disc degeneration was determined according to Schneiderman's classification for magnetic resonance imaging (MRI) [22]. The control sample consisted of 906 healthy check-up examinees who were matched to the cases by age and gender. They had no history of back problems and had negative MRI findings. Individuals with familial relation to any of the patients and a history of seeking medical attention for back pain or sciatica were excluded. All the subjects were unrelated Han Chinese.

This study was approved by the ethics committee of Qi Lu Hospital, Shan Dong Province, China. The ethics committee approved data collection of these patients. All subjects signed a written informed consent form. All works were undertaken following the provisions of the Declaration of Helsinki.

\section{Genotyping}

Genomic DNA from all the subjects was extracted from peripheral blood leukocytes using a DNA isolation kit following the manufacturer's instructions (Invitrogen, USA).

To determine the polymorphism distribution of patients and controls concerning the two SNPS $(-378 \mathrm{~T} / \mathrm{C}$ and $-364 \mathrm{G} / \mathrm{T})$, a $495 \mathrm{bp}$ fragment of the human MT1-MMP 5' regulatory region spanning $b p-585$ to -90 was amplified by polymerase chain reaction (PCR) using the BD Advantage 2 PCR System (Takara BIO, Potsdam) and the flanking primer pair 5'-GCCACATAGCCCCCAATAAT-3' 
and 5'-AGATCTTTGTCTTCGGTA-3'. Polymerase chain reaction was carried out with $1 \mu$ of genomic DNA from controls and patients, respectively, with the following protocol: 2 min at $95^{\circ} \mathrm{C}$ followed by 35 cycles $\left(30 \mathrm{~s}\right.$ at $95^{\circ} \mathrm{C}, 30 \mathrm{~s}$ at $61^{\circ} \mathrm{C}, 40 \mathrm{~s}$ at $68^{\circ} \mathrm{C}$ ) and a final $1 \mathrm{~min}$ at $68^{\circ} \mathrm{C}$. Polymerase chain reaction products were purified and sequenced with a nested primer 5'-GAACTGGGGTCTGGTT-3' in high throughput 96-well plates using a BigDye Terminator v3.1 Cycle Sequencing kit (Applied Biosystems, Foster City) on an ABI3730 automated DNA sequencer. Allelic frequencies and Hardy-Weinberg distributions were calculated using standard methodologies.

Positive and negative controls were used in each genotyping assay, and 5\% of the samples were randomly selected and run in duplicate with $100 \%$ concordance. The results were reproducible with no discrepancy in genotyping.

\section{Statistical analysis}

Statistical analysis were performed using SPSS version 19.0 software. The characteristics of IVDD patients and controls were compared with the $\chi^{2}$ or Student's $t$ test according to the variable types. The $\chi^{2}$ test was performed to assess Hardy-Weinberg equilibrium. Comparison of genotype and allele frequencies between cases and controls was carried out in terms of the $\chi^{2}$ test. The risk asso- ciated with individual alleles and genotypes was calculated as the odds ratio (OR) with $95 \%$ confidence intervals $(\mathrm{Cl})$ using unconditional logistic regression. The severity of disc degeneration of different genotypes among IVDD patients was analyzed using the $\chi^{2}$ test. All statistical tests were two-sided, and $p<0.05$ for the differences was considered statistically significant.

\section{Results}

\section{Baseline parameters}

No significant differences in age and gender were found between IVDD patients and normal controls, but the body mass index (BMI) of patients was a little higher than healthy subjects; this suggested that obesity was a risk factor for IVDD. The characteristics of the two groups are presented in Table I.

Association between -378T/C and -364 G/T polymorphisms of the MMP14 gene and the risk of intervertebral disc degeneration

The genotype and allele distributions of the $-378 \mathrm{~T} / \mathrm{C}$ and $-364 \mathrm{G} / \mathrm{T}$ polymorphisms of the MMP14 gene in the IVDD and control groups are shown in Table II. The genotype distribution of the two polymorphisms in controls was in with the Hardy-Weinberg equilibrium ( $p=0.078$ for $-378 \mathrm{~T} / \mathrm{C}$,

Table I. Characteristics of patients with IVDD and healthy controls

\begin{tabular}{|lccc|}
\hline Characteristics & Patients with IVDD & Healthy controls & Value of $p$ \\
\hline Age [years] & $51.12 \pm 7.65$ & $51.54 \pm 8.35$ & 0.504 \\
\hline Gender (male/female) & $552 / 356$ & $555 / 351$ & 0.469 \\
\hline BMI (means \pm SD) & $23.5 \pm 2.2$ & $23.2 \pm 2.5$ & $<0.01$ \\
\hline
\end{tabular}

Table II. Genotype and allele distributions of $-378 \mathrm{~T} / \mathrm{C}$ and $-364 \mathrm{G} / \mathrm{T}$ polymorphisms in the IVDD and control groups

\begin{tabular}{|c|c|c|c|c|c|c|c|c|}
\hline \multirow[t]{2}{*}{ Location } & \multirow[t]{2}{*}{ Genotype } & \multicolumn{2}{|c|}{ IVDD group } & \multicolumn{2}{|c|}{ Control group } & \multirow[t]{2}{*}{ Value of $p^{*}$} & \multirow[t]{2}{*}{ OR $(95 \% \mathrm{Cl})$} & \multirow[t]{2}{*}{ Value of $p^{\#}$} \\
\hline & & $n$ & $\%$ & $n$ & $\%$ & & & \\
\hline \multirow[t]{5}{*}{-378} & $\mathrm{TT}$ & 370 & 41.3 & 294 & 32.5 & 0.001 & 1 & \\
\hline & CT & 387 & 40.2 & 421 & 46.5 & & $0.730(0.594-0.898)$ & 0.003 \\
\hline & CC & 151 & 18.5 & 191 & 21.0 & & $0.628(0.483-0.817)$ & 0.001 \\
\hline & T & 1127 & 62.1 & 1009 & 55.7 & $<0.001$ & 1 & \\
\hline & $C$ & 689 & 37.9 & 803 & 44.3 & & $0.768(0.673-0.877)$ & $<0.001$ \\
\hline \multirow[t]{5}{*}{-364} & GG & 405 & 44.6 & 409 & 45.2 & 0.496 & 1 & \\
\hline & GT & 408 & 44.9 & 417 & 46.0 & & $0.988(0.814-1.199)$ & 0.903 \\
\hline & $\mathrm{TT}$ & 95 & 10.5 & 80 & 8.8 & & $1.199(0.864-1.664)$ & 0.277 \\
\hline & G & 1218 & 67.1 & 1250 & 68.2 & 0.458 & 1 & \\
\hline & $\mathrm{T}$ & 598 & 32.9 & 582 & 31.8 & & $1.055(0.918-1.212)$ & 0.454 \\
\hline
\end{tabular}

${ }^{\star} P$ value was calculated by $\chi^{2}$ test among all the different genotypes; ${ }^{\#} p$ value was calculated by unconditional logistic regression. 
and $p=0.069$ for $-364 \mathrm{G} / \mathrm{T})$. There were significantly lower frequencies of the $-735 \mathrm{CT}$ and CC genotypes in patients with IVDD compared with normal controls $(p<0.001)$. Patients with IVDD showed a significantly lower frequency of the $C$ allele than normal controls $(p<0.001)$. Unconditional logistic regression analysis revealed that the $C$ allele was significantly associated with the decreased risk of IVDD compared with the T allele $(p<0.001$; OR $=0.768 ; 95 \% \mathrm{Cl}: 0.673-0.877)$, and CT and TT genotypes were significantly associated with a decreased risk of IVDD compared with the CC genotype (for CC genotype, $p<0.001$; $\mathrm{OR}=0.628 ; 95 \% \mathrm{Cl}: 0.483-0.817$; for $\mathrm{CT}$ genotype, $p=0.003, \mathrm{OR}=0.730,95 \% \mathrm{Cl}: 0.594-0.898$ ).

There is no significant correlation between $-364 \mathrm{G} / \mathrm{T}$ polymorphism and risk of IVDD (Table II).

\section{Association of $-378 \mathrm{~T} / \mathrm{C}$ polymorphism with the severity of intervertebral disc degeneration}

The association between the genotype and allele distribution of the $-378 \mathrm{~T} / \mathrm{C}$ polymorphism and the severity of IVDD is presented in Table III. The IVDD patients with the -378CC genotype showed significantly lower grades of disc degeneration compared with those with the -378TT genotype $(p<0.001)$. Although there was no significant difference in severity (from grade 2 to grade 4 ) in the $\mathrm{C}$ allele, compared to the $\mathrm{T}$ allele, the $\mathrm{C}$ allele had a higher ratio in grade 2 and a lower ratio in grade $4(p<0.001)$.

\section{Discussion}

The exact mechanism of IVDD is still unknown. Recent studies have focused on the role of genetic factors in the etiology of IVDD, and epidemiologic studies suggest that heredity is the largest single determinant of disc degeneration [23]. Biochemical mediators of tissue degradation, especially MMPs, have been identified as significant factors $[24,25]$. Previous studies demonstrated that the MMP family plays important roles in the pathology of IVDD. Our study has shown that the MMP14 promoter genotype was associated with IVDD degeneration in a large population of Han Chinese.
The frequency of the MMP14-378TT genotype was significantly higher in patients with IVDD than in the healthy population. Subjects with the TT genotype had a nearly 1.59-fold increased risk for IVDD compared to the CC genotype $(p<0.01)$. In addition, this genotype was found to correlate with more severe grades of disc degeneration observed on MRI.

Although the activity of MMP14 is known to be regulated by posttranscriptional mechanisms, inclusion of the activation of proenzyme and inhibition of TIMPS and transcriptional regulation also play a major role. Recently, Holmbeck et al. reported two closely functional SNPs of the MMP14 gene, $-378 \mathrm{~T} / \mathrm{C}$ and $-364 \mathrm{G} / \mathrm{T}$, both located at the RR1 region [11]. One $(-378 \mathrm{~T} / \mathrm{C})$ of the two SNPs flanking the RR1 was highly relevant for the transcriptional regulation of MMP14. While the wild type $(-378 \mathrm{~T})$ displayed a powerful enhancer function of RR1, the genetic variant (-378 C) leads to considerable loss in transcriptional activation (75\%). As the variant $-364 \mathrm{~T}$ revealed no significant reduction in transcriptional activation of MMP14, it was excluded from our further investigations. In our present study, consistent with known studies, MMP14 protein expression might be higher in individuals who carry the TT genotype than those who carry the CC or CT genotype at the -374 position, which showed higher risk of IVDD. While the -364 polymorphism did not show a significant influence on the altered risk of IVDD, we hypothesize that it is correlated with the different enhancement of transcriptional activation of two SNPs. $-378 T / C$ SNP could alter the transcriptional regulation of MMP-14 and contribute to the "over-expression" of MMP14 in IVDD.

Biochemically, disc degeneration is characterized by enhanced breakdown of the matrix. The main components of disc ECM, collagens and proteoglycans, are degraded by a specific class of proteolytic enzymes known as MMPs. Several lines of evidence show that MMPs play a pivotal role in regulation of intervertebral disc homeostasis. Unlike most MMP family members, MMP14 is expressed at the cell surface rather than secreted. One important role of MMP14 is to activate MMP2. A series of previous studies have demon-

Table III. Association of different genotypes and alleles of $-378 \mathrm{~T} / \mathrm{C}$ polymorphism with severity of IVDD

\begin{tabular}{|lccccc|}
\hline Genotype & $n$ & Grade 2 & Grade 3 & Grade 4 & Value of $p$ \\
\hline TT & 370 & $59(15.9 \%)$ & $93(25.1 \%)$ & $218(58.9 \%)$ & $<0.001$ \\
\hline CT & 387 & $78(20.2 \%)$ & $112(28.9 \%)$ & $27(50.9 \%)$ & $<.9 \%)$ \\
\hline CC & 151 & $76(50.3 \%)$ & $48(31.8 \%)$ & $633(56.2 \%)$ & $251(36.4 \%)$ \\
\hline T & 1127 & $196(17.4 \%)$ & $298(26.4 \%)$ & $208(30.2 \%)$ & \\
\hline C & 689 & $230(33.4 \%)$ & & & \\
\hline
\end{tabular}


strated that expression and activity of MMP2 is associated with increased risk of disc degeneration disease [26-28]. We hypothesize that overexpression of MMP14 increases the relative risk of IVDD by over-activating MMP2 expression. The studied polymorphism might not be the direct cause of IVDD, but could instead be a genetic marker that is in linkage disequilibrium with a true disease predisposing a locus nearby. Intervertebral disc degeneration was associated with the activity of MMP2, and the correlation of its activation with MMP14 production suggests that MMP14 activates MMP2 during disc degeneration.

Currently, no study regarding the relationship between the SNPS of MMP 14 and IVDD has been reported. But some studies have shown that MMP14 is involved in the pathology of IVDD. Apart from its role in activating other MMPs such as MMP2 and MMP13, MMP14 also has the capacity to degrade extracellular matrix components directly [29]. MMP14 itself is capable of matrix degradation, and in addition is capable of activating not only MMP2 but also MMP13, another MMP related to IVDD and MMP8 [30-32], enabling efficient and fast degradation of the resident matrix. Osteoarthritic degeneration of articular cartilage, a process that shares many similarities with IVDD [33], is associated with an increase in production of these four MMPs [34-36], which possibly renders MMP14 a key player in initiating and maintaining extracellular matrix destruction.

Upregulation of specific MMPs (MMP1, -2, -3, $-7,-8,-10$, and -13 ) was reported in human IVDD [37-39]. However, it is still unclear due to conflicting published studies. Numerous studies indicate that the expression levels of MMP are modulated by a combination of many factors, including mechanical, inflammatory, and oxidative stress. Genetic predisposition also plays an important role in determining gene expression of MMP1, -2, -3, and -9 . So upregulation of MMP expression and enzymatic activity is implicated in disc ECM destruction, leading to the development of IVDD [37, 40]. Future IVDD therapeutics depends on identifying specific MMPs whose dysregulation results in pathological proteolysis of disc ECM. In the current study, we also provide some evidence about the role of MMP14 in the development of IVDD.

There are also some drawbacks in the present study. One of them is that because all the subjects are Chinese individuals, the results should be interpreted with caution and need to be confirmed in larger and ethnically divergent population samples.

In conclusion, we found that MMP14-735C/T polymorphism may be a genetic risk factor associated with susceptibility to IVDD in Han Chinese individuals. The relation between the mutation of this gene and IVDD warrants further investigation.

\section{Conflict of interest}

The authors declare no conflict of interest.

\section{References}

1. Tan H, Zhao J, Jiang J, Ren Y. Association of the polymorphism of DR4 with the risk and severity of lumbar disc degeneration in the Chinese Han population. Scand J Clin Lab Invest 2012; 72: 576-9.

2. Katz JN. Lumbar disc disorders and low-back pain: socioeconomic factors and consequences. J Bone Joint Surg Am 2006; 88 Suppl 2: 21-4.

3. Higashino K, Matsui Y, Yagi S, et al. The alpha2 type IX collagen tryptophan polymorphism is associated with the severity of disc degeneration in younger patients with herniated nucleus pulposus of the lumbar spine. Int Orthop 2007; 31: 107-11.

4. Gruber HE, Hoelscher GL, Ingram JA, Bethea S, Zinchenko N, Hanley EN Jr. Variations in aggrecan localization and gene expression patterns characterize increasing stages of human intervertebral disk degeneration. Exp Mol Pathol 2011; 91: 534-9.

5. Mashayekhi F, Shafiee G, Kazemi M, Dolati P. Lumbar disk degeneration disease and aggrecan gene polymorphism in northern Iran. Biochem Genet 2010; 48: 684-9.

6. Lee JM, Song JY, Baek M, et al. Interleukin-1 beta induces angiogenesis and innervation in human intervertebral disc degeneration. J Orthop Res 2011; 29: 265-9.

7. Xu G, Mei Q, Zhou D, Wu J, Han L. Vitamin D receptor gene and aggrecan gene polymorphisms and the risk of intervertebral disc degeneration - a meta-analysis. PloS One 2012; 7: e50243.

8. Zigouris A, Alexiou GA, Batistatou A, Voulgaris S, Kyritsis AP. The role of matrix metalloproteinase 9 in intervertebral disc degeneration. J Clin Neurosci 2011; 18: 1424-5.

9. Yuan HY, Tang Y, Liang YX, et al. Matrix metalloproteinase-3 and vitamin D receptor genetic polymorphisms, and their interactions with occupational exposure in lumbar disc degeneration. J Occup Health 2010; 52: 23-30.

10. Mayer JE, latridis JC, Chan D, Qureshi SA, Gottesman O, Hecht AC. Genetic polymorphisms associated with intervertebral disc degeneration. Spine J 2013; 13: 299-317.

11. Munkert A, Helmchen U, Kemper MJ, Bubenheim M, Stahl RA, Harendza S. Characterization of the transcriptional regulation of the human MT1-MMP gene and association of risk reduction for focal-segmental glomerulosclerosis with two functional promoter SNPs. Nephrology Dialysis Transplantation 2009; 24: 735-42.

12. Lee H, Overall CM, McCulloch CA, Sodek J. A critical role for the membrane-type 1 matrix metalloproteinase in collagen phagocytosis. Mol Biol Cell 2006; 17: 4812-26.

13. Rutges JP, Kummer JA, Oner FC, et al. Increased MMP-2 activity during intervertebral disc degeneration is correlated to MMP-14 levels. J Pathol 2008; 214: 523-30.

14. Chen TY, Li YC, Liu YF, et al. Role of MMP14 gene polymorphisms in susceptibility and pathological development to hepatocellular carcinoma. Ann Surg Oncol 2011; 18: 2348-56.

15. Itoh Y, Seiki M. MT1-MMP: a potent modifier of pericellular microenvironment. J Cell Physiol 2006; 206: 1-8.

16. Ohnishi K, Takagi M, Kurokawa Y, Satomi S, Konttinen YT. Matrix metalloproteinase-mediated extracellular matrix protein degradation in human pulmonary emphysema. Laboratory Investig 1998; 78: 1077-87. 
17. Jo Y, Yeon J, Kim HJ, Lee ST. Analysis of tissue inhibitor of metalloproteinases-2 effect on pro-matrix metalloproteinase- 2 activation by membrane-type 1 matrix metalloproteinase using baculovirus/insect-cell expression system. Biochem J 2000; 345: 511-9.

18. Zwierzchowski TJ, Stasikowska-Kanicka O, Janus J, Konecki W, Danilewicz M, Fabis J. Assessment of apoptosis, MMP-1, MMP-3, TIMP-2 expression and mechanical and biochemical properties of fresh rabbit's medial meniscus stored two weeks under tissue culture conditions. Arch Med Sci AMS 2014; 10: 167-73.

19. Seguin CA, Pilliar RM, Madri JA, Kandel RA. TNF-alpha induces MMP2 gelatinase activity and MT1-MMP expression in an in vitro model of nucleus pulposus tissue degeneration. Spine 2008; 33: 356-65.

20. Dong DM, Yao M, Liu B, Sun CY, Jiang YQ, Wang YS. Association between the $-1306 \mathrm{C} / \mathrm{T}$ polymorphism of matrix metalloproteinase- 2 gene and lumbar disc disease in Chinese young adults. Eur Spine J 2007; 16: 1958-61.

21. Zhang Y, Gu Z, Qiu G. Association of the polymorphism of MMP2 with the risk and severity of lumbar disc degeneration in the Chinese Han population. Eur Rev Med Pharmacol Sci 2013; 17: 1830-4.

22. Schneiderman G, Flannigan B, Kingston S, Thomas J, Dillin WH, Watkins RG. Magnetic resonance imaging in the diagnosis of disc degeneration: correlation with discography. Spine 1987; 12: 276-81.

23. Sambrook PN, MacGregor AJ, Spector TD. Genetic influences on cervical and lumbar disc degeneration: a magnetic resonance imaging study in twins. Arthritis Rheumatism 1999; 42: 366-72.

24. Goupille P, Jayson MI, Valat JP, Freemont AJ. Matrix metalloproteinases: the clue to intervertebral disc degeneration? Spine 1998; 23: 1612-26.

25. Takahashi M, Haro H, Wakabayashi Y, Kawa-uchi T, Komori $\mathrm{H}$, Shinomiya $\mathrm{K}$. The association of degeneration of the intervertebral disc with $5 \mathrm{a} / 6 \mathrm{a}$ polymorphism in the promoter of the human matrix metalloproteinase-3 gene. J Bone Joint Surgery 2001; 83: 491-5.

26. Roberts S, Caterson B, Menage J, Evans EH, Jaffray DC, Eisenstein SM. Matrix metalloproteinases and aggrecanase: their role in disorders of the human intervertebral disc. Spine 2000; 25: 3005-13.

27. Vaccaro AR, Carrino JA, Venger BH, et al. Use of a bioabsorbable anterior cervical plate in the treatment of cervical degenerative and traumatic disc disruption. J Neurosurgery 2002; 97: 473-480.

28. Weiler C, Nerlich AG, Zipperer J, Bachmeier BE, Boos N. 2002 SSE Award Competition in Basic Science: expression of major matrix metalloproteinases is associated with intervertebral disc degradation and resorption. Eur Spine J 2002; 11: 308-20.

29. Holmbeck K, Bianco P, Birkedal-Hansen H. MT1-MMP. a collagenase essential for tumor cell invasive growth. Cancer Cell 2003; 4: 83-4.

30. Roberts S, Evans H, Trivedi J, Menage J. Histology and pathology of the human intervertebral disc. J Bone Joint Surg Am 2006; 88 Suppl 2: 10-4.

31. Holopainen JM, Moilanen JA, Sorsa T, et al. Activation of matrix metalloproteinase- 8 by membrane type 1-MMP and their expression in human tears after photorefractive keratectomy. Investig Ophthalmol Visual Sci 2003; 44: 2550-6.

32. Murphy G, Knauper V, Atkinson S, et al. Matrix metalloproteinases in arthritic disease. Arthritis Res 2002; 4 Suppl 3: S39-49.

33. Mwale F, Roughley P, Antoniou J. Distinction between the extracellular matrix of the nucleus pulposus and hyaline cartilage: a requisite for tissue engineering of intervertebral disc. Eur Cell Mater 2004; 8: 58-63; discussion 63-54.

34. Bramono DS, Richmond JC, Weitzel PP, Kaplan DL, Altman $\mathrm{GH}$. Matrix metalloproteinases and their clinical applications in orthopaedics. Clin Orthop Relat Res 2004; 428: 272-85.

35. Duerr S, Stremme S, Soeder S, Bau B, Aigner T. MMP-2/ gelatinase $A$ is a gene product of human adult articular chondrocytes and is increased in osteoarthritic cartilage. Clin Exp Rheumatol 2004; 22: 603-8.

36. Tetlow LC, Adlam DJ, Woolley DE. Matrix metalloproteinase and proinflammatory cytokine production by chondrocytes of human osteoarthritic cartilage: associations with degenerative changes. Arthritis Rheumatism 2001; 44: 585-94.

37. Loreto C, Leonardi R, Musumeci G, Pannone G, Castorina S. An ex vivo study on immunohistochemical localization of MMP-7 and MMP-9 in temporomandibular joint discs with internal derangement. Eur J Histochem 2013; 57: e12.

38. Vo NV, Hartman RA, Yurube T, Jacobs LJ, Sowa GA Kang JD. Expression and regulation of metalloproteinases and their inhibitors in intervertebral disc aging and degeneration. Spine J 2013; 13: 331-41.

39. Yurube T, Takada T, Suzuki T, et al. Rat tail static compression model mimics extracellular matrix metabolic imbalances of matrix metalloproteinases, aggrecanases, and tissue inhibitors of metalloproteinases in intervertebral disc degeneration. Arthritis Res Ther 2012; 14: R51.

40. Jin ES, Min J, Jeon SR, Choi KH, Jeong JH. Analysis of molecular expression in adipose tissue-derived mesenchymal stem cells: prospects for use in the treatment of intervertebral disc degeneration. J Korean Neurosurg Soc 2013; 53: 207-12 